\title{
Comparing load balancing algorithms for web application in cloud environment
}

\author{
Zakaria Benlalia ${ }^{1}$, Karim Abouelmehdi ${ }^{2}$, Abderrahim Beni-hssane ${ }^{3}$, Abdellah Ezzati ${ }^{4}$ \\ 1,2,3 LAROSERI laboratory, Computer Science Department, Sciences Faculty, Chouaïb Doukkali University, Morocco \\ ${ }^{4}$ LAVETE laboratory, Sciences and Technics Faculty, Hassan 1st University, Morocco
}

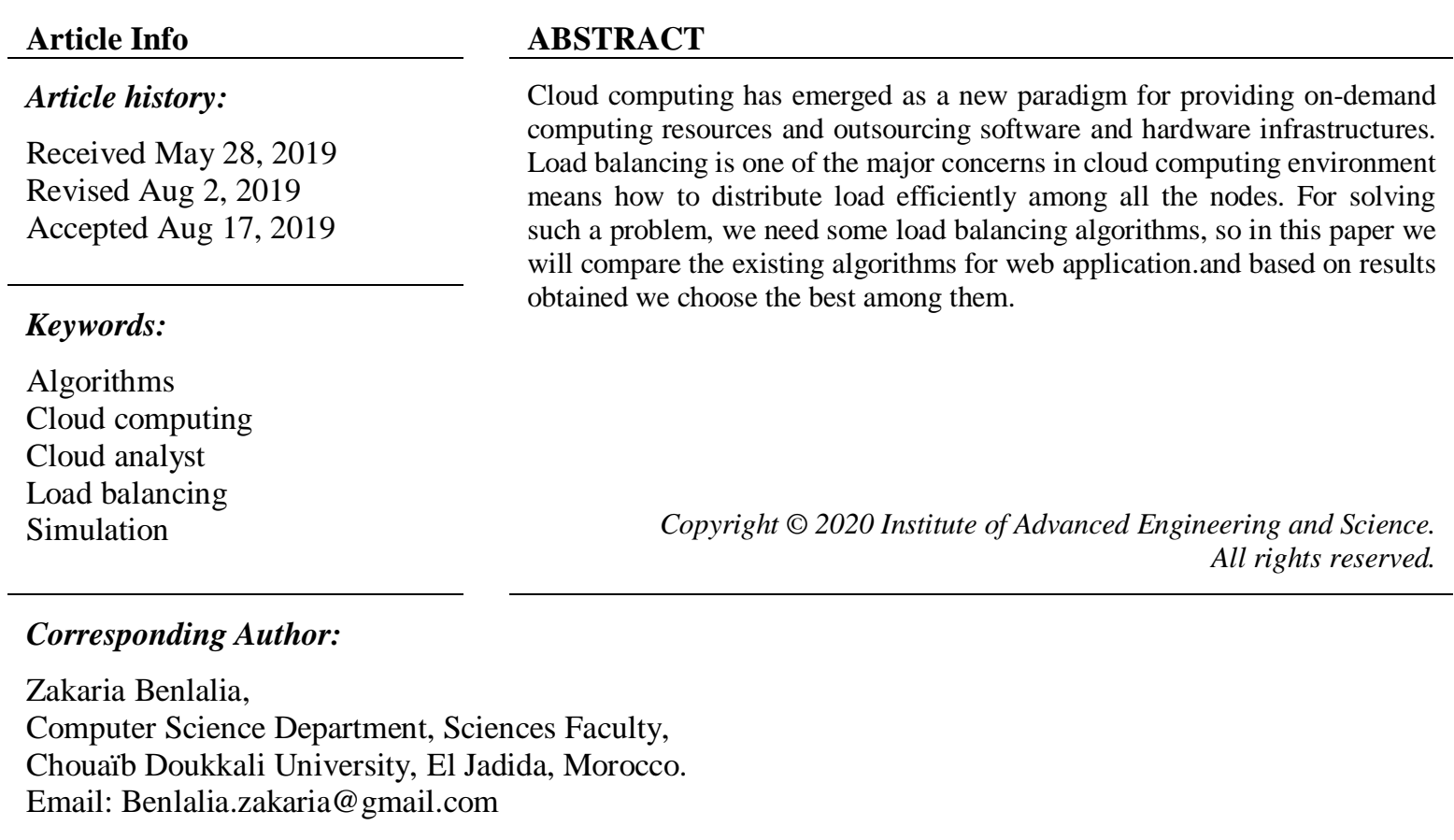

\section{INTRODUCTION}

Cloud computing progress open up many new opportunities for developers of Internet applications [1]. Cloud computing offers distributed and shared computing resources and services that belong to different service providers and websites [2], thanks to models and designs that make infrastructures, platforms and softwares as well as services [3].

Cloud computing is a large-scale distributed computing paradigm that is driven by economies of scale, in which a pool of virtualized, abstracted, and dynamically scalable managed computing power, platforms, storage, and services are delivered on demand to external customers over the Internet [4]. Standardizing the definition of cloud computing provided by The National Institute of Standards and Technology (NIST) [5]. NIST definition of cloud computing Cloud computing is a model for enabling convenient, on-demand network access to a shared pool of configurable computing resources like servers, networks, storage, applications etc., that can be rapidly provisioned and released with minimal management effort or service provider interaction.Cloud computing is not an innovative technology, but rather a new operations model that carries together a set of present technologies to run business in an innovative way [6].

Data center-based cloud infrastructure in the form of physical servers includes virtualization techniques [7], to maximize utilization and increase the overall performance of the architecture. Task processing and load balancing between virtual machines in a data center are performed using load balancing algorithms, static and dynamic $[8,9]$, under the supervision of a control center controller data.

The complexity of the cloud infrastructure, the weaknesses of load balancing algorithms, and the variety of stakeholders to execute client requests put cloud service providers ahead of the quality of service challenges $[10,11]$. Among the big challenge to cloud computing is security $[12,13]$, and load ballancing. 
In this paper, we study the literature of the load-balancing algorithms and present a comparison of load balancing algorithms existing in cloud analyst to show the importance of selection of the load balanced algorithm of a web application, which will be hosted in a cloud environment in several geographical areas, before choosing the cloud provider

\section{LOAD BALANCING ALGORITHMS}

Load balancing is an important issue in networks. Load balancing of request distributes workloads across multiple computing resources, such as computers, computer cluster, disk drives, network links, CPU. The load can be balanced dynamically or statically. In some situations, the load on a particular node cannot be foreseen. This remains a key issue specifically in distributed networks. Load Balancingis a method of dispensing the requests to many servers or resources. This helps in enhancing the performance, efficient utilization of resources, avoids overloading, reduced response time and improved throughput [14].

The problem of load balancing as a persisting old problem ; as a result, many approaches have been proposed to solve this issue.

a) Round Robin Algorithm [15]: The algorithm works on random selection of the virtual machines. The datacenter controller assigns the requests to a list of VMs on a rotating basis. The first request is allocated to a VM picked randomly from the group and then the Data Center controller assigns the requests in a circular order. Once the VM is assigned the request, the VM moves to the end of the list [16].

b) Equally Spread Current Execution : This algorithm processes to address priorities [17]. In other words, the algorithm dispenses the load randomly by analyzing the volume and relocating the load to those virtual machines that are lightly loaded or need to handle this task easily, need less time to complete the task and maximize throughput. The tasks are transferred to the VM manager, which maintains a set of tasks and their necessary resources.

c) Throttled load balancer: This algorithm is entirely based on the virtual machine. with this algorithm, the user first communicates with the load balancer to find a suitable virtual machine for the required operation [18]. If a suitable machine is identified, the load balancer accepts the client request and assigns that virtual machine to the client. Otherwise, the request is queued.

The objective veral geographic areas, by assessing, with CloudAnalyst simulator, the response time and Data Center processing of our study is to show the simplicity of using cloud technology to host a largescale web application in se time. And then make decision of the best load balanced algorithm.

Load balancing is a crucial prominence in cloud computing, especially the hybrid clouds in order to cope with the service level agreement (SLA) and the quality of service (QoS) [19]. Any algorithm for load balancing is designed, depending on the state or behavior of the system, which may be static or dynamic [20].

a) Static algorithms [21]: These algorithms do not depend on the current state of the system and have a prior knowledge of system resources and details of all the tasks of an application. These types of algorithms face a major disadvantage in the event of a sudden failure of system resources and tasks.

b) Dynamic algorithms [22]: These algorithms make decisions about load balancing based on the current state of the system and do not require any prior knowledge of the system. This approach is an improvement over the static. The algorithms in this category are considered complex, but they have better fault tolerance and better performance.

\section{A COMPARATIVE SIMULATION OF LOAD BALANCING ALGORITHMS FOR A SOCIAL NETWORK APPLICATION}

In this case study that is made by the simulator Cloud Analyst [23, 24] we worked with data from social network application "Facebook" on which we will calculate the the Data Center processing time. For each region, we used several scenarios. We will work with the data found in 2012 with a scale of 1/1000. This application is accessed by users from all around the world. Asia (2678100 users), North America (2365200 users), South America (1444000 users), Europe (2499900 users), Oceania (146200 users) and Africa $(504,300$ users).

\subsection{Configuring the Simulation}

Based on the above information, we set for the number of users each user group was approximately $10 \%$ of all users during peak hours, the number of users accounting for nonpeak hours to $10 \%$ of peak time users. The detailed settings are showed in Table 1. And we assume the Americas, Europe, Oceania user requests once every five minutes; Asia users request once every three minutes; Africa once the user requests every 10 minutes. The user requests data size is $100 \mathrm{~b}$ each time. Cloud resource services agency policy is the 
closed data center policies. Data center contains 100 identically configured servers, each with the x86 architecture, Linux operating system, $2 \mathrm{G}$ memory, $100 \mathrm{G}$ storage spaces, and four processors. And the processor speed is 10000MIPS; Bandwidth is 1000Mbps; Virtual machine resource scheduling policy is the time-sharing. Applications in the experiments virtual local storage size is $100 \mathrm{MB}$, the virtual machine has $1 \mathrm{~GB}$ of RAM and $10 \mathrm{MB}$ of available bandwidth. Users are grouped by a factor of 1000, and requests are grouped by a factor of 100 . Each user request requires 250 instructions to be executed. Service broker policy is Closest Data center. In terms of the cost of hosting applications in a Cloud, we assume a pricing plan which closely follows the actual pricing plan of Amazon EC2. The assumed plan is: Cost per VM per hour (1024Mb, 100MIPS): \$ 0.10; Cost per 1 GB of data transfer (from/to Internet):\$0.10 [25].

Table 1. User Bases Used in the Experiment

\begin{tabular}{ccccc}
\hline User Base & Region & Peak Hours & Online User During Peak HRS & Online User During Off-Peak HRS \\
\cline { 2 - 5 } UB1 & N.Ameria & $3: 00 \sim 9: 00$ & 236520 & 23652 \\
UB2 & S.America & $3: 00 \sim 9: 00$ & 144400 & 14440 \\
UB3 & Europe & $3: 00 \sim 9: 00$ & 249990 & 24999 \\
UB4 & Asia & $3: 00 \sim 9: 00$ & 267810 & 26781 \\
UB5 & Africa & $3: 00 \sim 9: 00$ & 50430 & 5043 \\
UB6 & Oceania & $3: 00 \sim 9: 00$ & 14620 & 1462 \\
\hline
\end{tabular}

\subsection{Simulated Scenarios}

Several scenarios are considered in our case study. The easiest one is to model the case in which only one centralized data center is used to host the application of social network "Facebook". In this model, all requests of all users across the globe are processed by this single data center. A data center has 40 virtual machines assigned to the application is located in North America. Data entry as shown in Figure 1. Result of the first scenario as shown in Figure 2.

The second scenario consists to use two data centers, each one of them has 20 virtual machines dedicated to the application and they are located in Europe and North America.

In the last scenario, three data centers each contains the following number of virtual machines $(10,40$, and 20) are also located in North America, Europe and Asia.

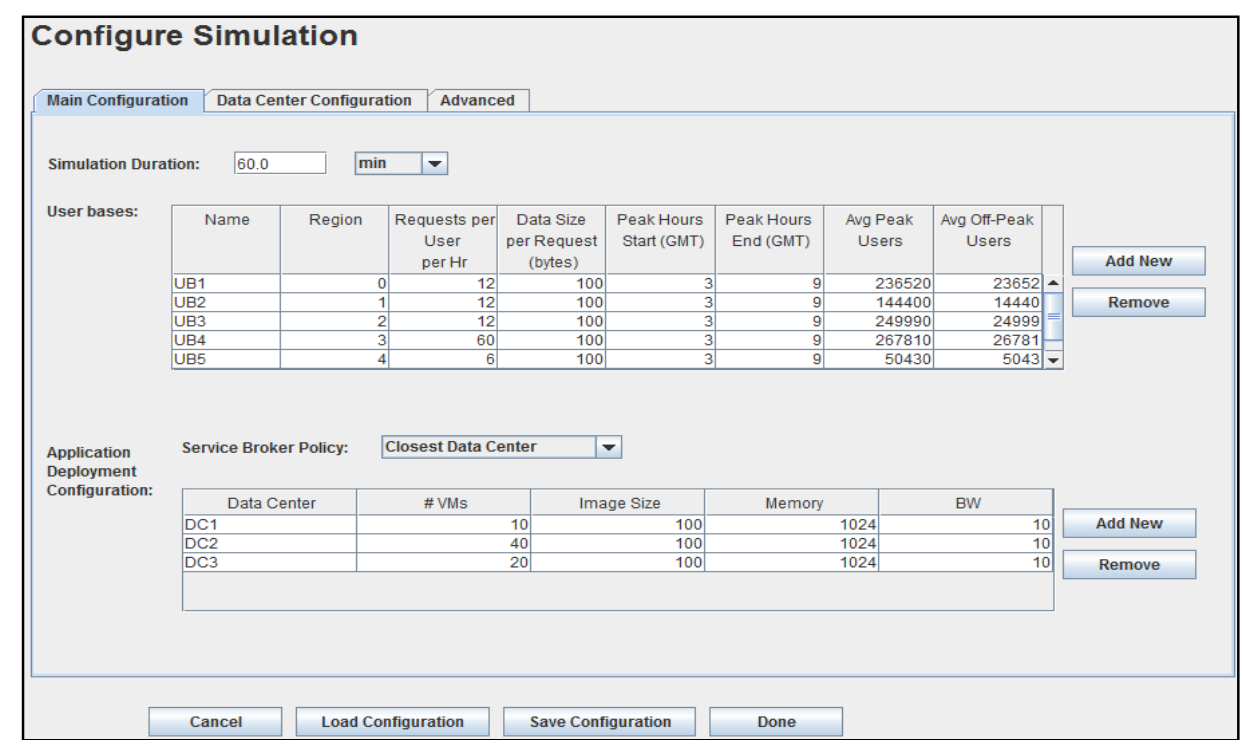

Figure 1. Data entry 


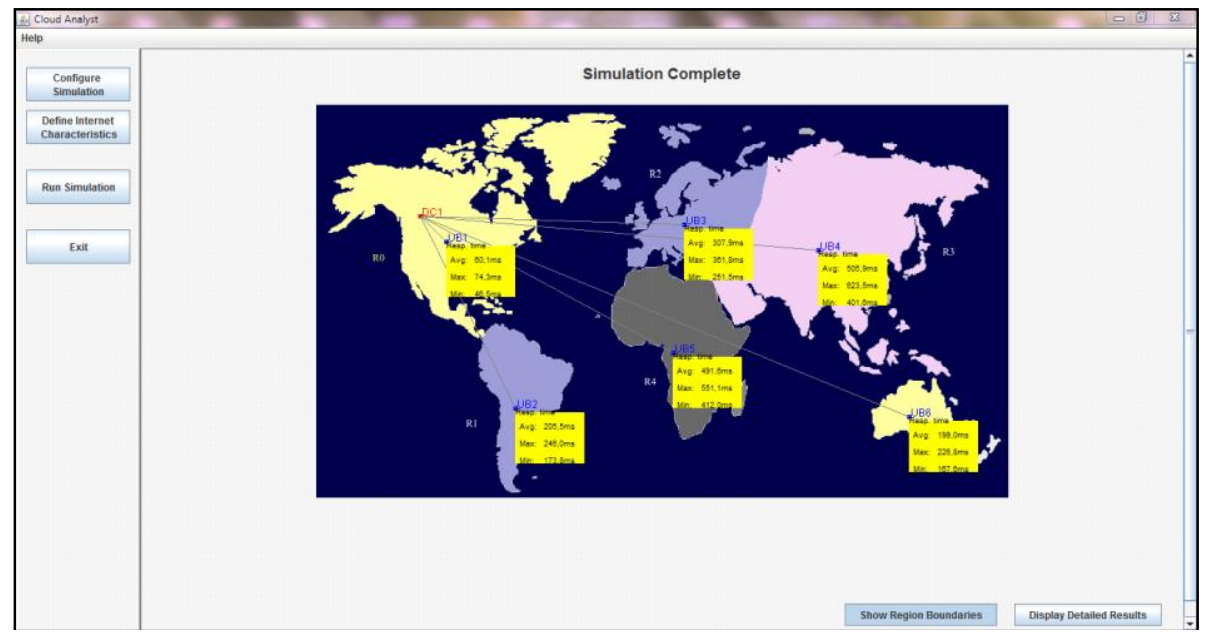

Figure 2. Result of the first scenario

\subsection{Results}

Simulation results for each Senario as shown in Table 2.

Table 2. Simulation Results for Each Senario

\begin{tabular}{cccc}
\hline Scenario & Load Balancer Algorithm & Overall response time & Data Center processing time \\
\hline \multirow{2}{*}{ First Scenario } & Round Robin & 401,32 & 3,74 \\
& Equally spread current execution load & 401,27 & 3,73 \\
& Throttled & 400,9 & 3,31 \\
\multirow{2}{*}{ Second Scenario } & Round Robin & 242,59 & 9,51 \\
& Equally spread current execution load & 242,33 & 9,19 \\
\multirow{2}{*}{ Third Scenario } & Throttled & 239,09 & 5,97 \\
& Equally spread current execution load & 234,56 & 12,92 \\
& Round Robin & 234,21 & 12,61 \\
\hline
\end{tabular}

\subsection{Discussion of Results}

The results are shown in Figure 3. After this comparison of the default load balancing algorithms in cloud analyst, we find out that the best load balanced algorithm is Throttled.

= Overall response time $\quad$ Data Center processing time

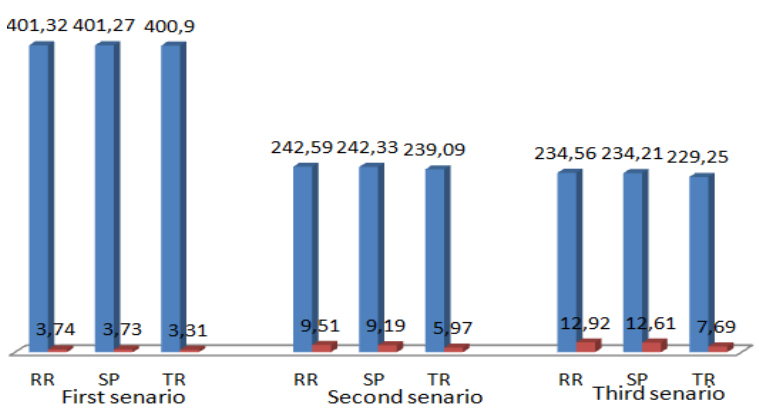

Figure 3. Results comparison

\section{CONCLUSION}

Cloud Computing is a huge concept and load balancing plays a very important role in Clouds. To the best of our knowledge in load balancing concept, once simulator with three default load balancing policy were compared with different parameters such as response time and Data Center processing time that 
were discussed beforehand. Through this comparison we show that the Throttled is the best load balanced algorithm. In the future work, we will try to improve the Throttled algorithm and compare it with the rest of the load balancer algorithms to make sure of its effectiveness.

\section{REFERENCES}

[1] Abouelmehdi, K., Beni-hssane, A., Benlalia, Z., Ezzati, A., \& Moutaouikkil, A. (2016). Assessing Cost and Response Time of a Web Application Hosted in a Cloud Environment. Europe and MENA Cooperation Advances in Information and Communication Technologies, 451-457. doi:10.1007/978-3-319-46568-5_46

[2] N.Chandrakala,B.Thirumala Rao" Migration of Virtual Machine to improve the Security in Cloud Computing", International Journal of Electrical and Computer Engineering (IJECE), Volume 8 No 2, February 2018.

[3] Akhil Goyal,Bharti Mohali," A Study of Load Balancing in Cloud Computing using Soft Computing Techniques", International Journal of Computer Applications (0975 - 8887) Volume 92 - No.9, April 2014.

[4] Benlalia, Z, Beni-hssane, A, Abouelmehdi, K" A new service broker algorithm optimizing the cost and response time for cloud computing" Procedia Computer Science 151 (2019) 992-997.

[5] NIST Definition of Cloud Computing v15, csrc.nist.gov/groups/SNS/cloud-computing/cloud-defv15.doc

[6] Qi Zhang, Lu Cheng, Raouf Boutaba. "Cloud computing: state-of-the-art and research challenges", J Internet ServAppl (2010) 1: 7-18, Springer

[7] Chaima Ghribi, Makhlouf Hadji, and Djamal Zeghlache. Energy efficient vm scheduling for cloud data centers: Exact allocation and migration algorithms. In CCGRID, pages 671 \{678. IEEE Computer Society, 2013. ISBN 9781-4673-6465-2.

[8] Ali M. Alakeel, “A Guide to Dynamic Load Balancing in Distributed Computer Systems", IJCSNS International Journal of Computer Science and Network Security, Vol.10 No.6, June 2010.

[9] Ram Prasad Padhy (107CS046), PGoutam Prasad Rao (107CS039).”Load balancing in cloud computing system" Department of Computer Science and Engineering National Institute of Technology, Rourkela Rourkela-769 008, Orissa, India May, 2011.

[10] Doddini Probhuling,"Load Balancing Algorithms in Cloud Computing", International Journal of Advanced Computer and Mathematical Sciences, ISSN 2230-9624. Vol 4, Issue3, pp (229-233), March 2013.

[11] Sakthivelmurugan V, Saraswathi A, Shahana R, "Enhanced Load Balancing Technique in Public Cloud", IJREAT International Journal of Research in Engineering \& Advanced Technology, Volume 2, Issue 2, Apr-May, 2014.

[12] El Makkaoui, K., Ezzati, A., Beni-Hssane, A., \& Ouhmad, S. (2019). Fast Cloud-Paillier homomorphic schemes for protecting confidentiality of sensitive data in cloud computing. Journal of Ambient Intelligence and Humanized Computing. doi:10.1007/s12652-019-01366-3

[13] El Makkaoui, K., Beni-Hssane, A., \& Ezzati, A. (2018). Speedy Cloud-RSA homomorphic scheme for preserving data confidentiality in cloud computing. Journal of Ambient Intelligence and Humanized Computing. doi:10.1007/s12652-018-0844-x

[14] V.Anand,K.Anuradha,"A Prolific Scheme for Load Balancing Relying on Task Completion Time" International Journal of Electrical and Computer Engineering (IJECE), Volume 8, No 3, June 2018.

[15] Sotomayor, B., Montero, R. S., Llorente, I. M. \& Foster, I. (2009). Virtual infrastructure management in private and hybrid clouds. IEEE Internet Computing, 13(5), 14-22.

[16] Mr.Manan D. Shah, "Allocation Of Virtual Machines In Cloud Computing Using Load Balancing Algorithm” in International Journal of Computer Science and Information Technology \& Security (IJCSITS), ISSN: 2249-9555. Vol. 3, No.1, February 2013.

[17] Ms.Nitika, Ms.Shaveta, Mr. Gaurav Raj, "Comparative Analysis of Load Balancing Algorithms in Cloud Computing" in International Journal of Advanced Research in Computer Engineering \& Technology Volume 1, Issue 3, May 2012.

[18] B.Wickremasinghe, R.N.Calheiros,R.Buyya, Cloudanalyst: A cloudsimbased visual modeller for analysing cloud computing in: Proceedings of the 24th International Conference on Advanced Information Networking and Applications (AINA 2010), Perth, Australia,, 2010.

[19] Chanintorn Jittawiriyanukoon," Performance evaluation of proposed load balancing algorithm with unstable concurrent programs", Indonesian Journal of Electrical Engineering and Computer Science, Vol. 14, No. 3, June 2019.

[20] Sandip Patel," CloudAnalyst : A Survey of Load Balancing Policies", International Journal of Computer Applications · May 2015, DOI: 10.5120/20679-3525

[21] Shah, N., Farik, M.: Static load balancing algorithms in cloud computing: challenges \& solutions. Int. J. Sci. Technol. Res. 4(10), 365-367 (2015)

[22] Milani, A.S., Navimipour, N.J.: Load balancing mechanisms and techniques in the cloud environments: systematic literature review and future trends. J. Netw. Comput. Appl. 71, 86-98 (2016)

[23] Bhathiya, Wickremasinghe,"Cloud Analyst: A Cloud Sim-based Visual Modeller for Analysing Cloud Computing Environments and Applications", IEEE, 2010.

[24] Tanvee Ahmed, Yogendra Singh, "Analytic Study Of Load Balncing Techniques Using Tool Cloud Analyst". International Journal of Engineering Research and Applications. pp. 1027-1030, 2012.

[25] J. Gustedt, E. Jeannot, and Martin Quinson, "Experimental methodologies for large-scale systems: a survey," Parallel Processing Letters, vol. 19, Sep. 2009, pp. 399-418 